\title{
Comentario de libros
}

\section{Javier Vitoria Cormenzana, Instituto diocesano de Teología y Pastoral, Bilbao. Centro de Reflexión Teológica, San Salvador.}

Nota de la redacción. Las siguientes recensiones, hechas por Javier Vitoria, versan sobre libros que tratan temas afines y que, sobre todo, son recensionados desde la perspectiva de la teología de la liberación. Pueden ser leídas, pues, como una unidad y por ello las publicamos juntas.

A. González, Trinidad y Liberación. La teologia trinitaria considerada desde la perspectiva de la teología de la liberación, UCA Editores., Col. Teología Latinoamericana, San Salvador, 1994, 247 páginas.

Este libro es una joya y, consiguientemente, un valioso hallazgo. El autor realiza un ejercicio práctico de cómo las intuiciones centrales de la teología de la liberación implican necesariamente una nueva teología sistemática y la capacitan, por su originalidad y novedad, para dialogar con la teología europea. El tema elegido para su ensayo es la docrina trinitaria. Y creo que consigue brillantemente su objetivo.

El primer capítulo da cuenta con solvencia del estado de la cuestión del tema de la trinidad en la teología europea. Merece especial atención el apartado en el que señala los límites de la conceptualización moderna de la trinidad a pesar de haber corregido muchas de las deficiencias del modelo clásico. El uso teológico de los conceptos filosóficos de sustancia y de sujeto, tampoco superado por la teología política de J. Moltmann, aunque inicialmente los rechace, plantea la aporía de la doctrina trinitaria: afirmar la unidad a costa de la autonomía de las personas o ésta a costa de la unidad. Esta aporía se piensa que puede ser superada desde la perspectiva de la teología de la liberación.

A partir de aquí dedicará los tres capítulos siguientes a presentar el punto de partida de la teología de la liberación, el intento de L. Boff de hablar de la 
trinidad desde dicha perspectiva, y los rasgos fundamentales de la reflexión de la teología de la liberación sobre la trinidad a partir de la historia de la salvación. Del primero de ellos conviene retener su énfasis en el primado de la realidad frente al sentido o la verdad, que le permite destacar la prioridad de la realidad de la revelación frente a su verdad. Y esto significa señalar con vigor que la revelación de Dios ha acontecido en función de nuestra salvación. Dios no se ha manifestado primariamente como la verdad del mundo ni como el fundamento de toda verdad y de todo acontecimiento, sino en cuanto salvador, en el acto mismo de salvar a los pobres. Del segundo, su tomarse absolutamente en serio el valor soteriológico de la afirmación del dolor y el sufrimiento en Dios. Del tercero, su comprensión de la salvación como "deiformación" y de los pobres como "lugar soteriológico".

Los tres últimos capítulos están orientados a sacar consecuencias de la conclusión de los anteriores, para el intento teológico de formular lo que sea la trinidad inmanente en sí misma: la doctrina trinitaria es la formulación creyente de la experiencia de un Dios que se ha comprometido radicalmente y en su misma realidad con la historia humana por medio del Hijo y del Espíritu.

Para ello establecerá tres principios hermenéuticos: (a) la necesidad de pensar la trinidad en categorías históricas y no en las naturalistas, como ha sido lo característico de la historia de la teología. Tanto las relaciones de la personas como la monarquía del Padre han de entenderse primariamente desde la historia de la salvación, y más concretamente, como propugna la teología de la liberación, tal y como aparecen en la historia de Jesús. (b) En diálogo con W. Pannenberg muestra cómo, siempre que se entienda la trinidad económica fundamentalmente en términos de salvación y no de revelación, la tesis rahneriana de la identificación entre la trinidad económica y la inmanente dice que quien crea, libera y deiforma en la historia de la salvación no es sino la trinidad divina tal como es en sí misma, ya que ella no solamente "se da a conocer" en los procesos históricos, manteniéndose en su realidad distante de ellos, sino que los funda y asume en su realidad. (c) De acuerdo con el interés de la teología de la liberación de pensar la historia en términos de praxis social y desde la perspectiva del pobre, y tratando de ver cómo la comunión de las personas divinas fundamenta la unificación histórica y escatológica de la humanidad se encuentra con là categoría sociedad de esa teología. Releída zubirianamente, la sociedad no es ni sustancia ni sujeto, sino más bien estructura según la cual se configura la actividad de cada uno de sus miembros a partir de la primera socialización. Esto significa que ella no existe fuera de los hombres que la componen, ni que estos hombres sean puros momentos accidentales de una realidad sustancial. Al contrario, no hay sociedad fuera de los hombres que la integran, sino justamente en ellos. Y, en su virtud, la sociedad no es algo ulterior, sino esencial a su constitución como individuos y como personas. 
A partir de este momento está en condiciones de revisar críticamente el concepto de persona entendido como sustancia y como sujeto, y de llegar, haciendo un rodeo por Ricardo de San Víctor y gracias nuevamente a Zubiri, a un concepto de persona divina como realidad en constitutiva autoposesión dinámica, inseparable de su propia actividad. La esencia divina consistirá en la unidad de amor entre las mismas que se nos ha mostrado ya en la historia de la salvación. Inmanentemente, esto se expresa diciendo que la esencia divina es la perijóresis.

De esta manera, Dios es tres personas que se relacionan con el mundo como una comunidad, incluyéndolo en su comunión y conduciéndolo hacia una unidad que no es otra cosa que la participación en el amor en que consiste la vida divina. Dios es Amor y su relación con el mundo es primariamente una relación de inclusión, que explicita no simplemente las acciones externas de una sustancia o un sujeto que existe más allá de las mismas, sino efusivamente su misma realidad divina como actividad pura de amor.

Tras este recorrido está en condiciones de afirmar quelos pobres pertenecen a la definición de Dios. La presencia preferencial de Dios entre los pobres, manifestada en la historia de la salvación, no constituiría ni un mero acto pedagógico, ni tampoco un simple acto revelatorio, sino la efusión misma de la realidad trinitaria del Dios que es amor. Dios es afectado finitamente, humanamente por el dolor de los crucificados en su realidad misma en el Hijo, como amor anonadado, en El Espíritu, como dolor gimiente y resucitador en Jesús y en la historia, en el Padre, como ultimidad condoliente y solidaria.

Con esta obra, el autor se ha abierto un camino de diálogo teológico hasta ahora inexplorado: la doctrina trinitaria. $\mathrm{Y}$ lo ha hecho con una originalidad en su pensamiento y con un rigor y vigor epistemológico en su pensar, que estoy seguro va a sorprender. Ahora solamente queda esperar que, en lugar de repetir frases hechas sobre la entidad noética de la teología de la liberación, se acepte un ejercicio de la comunicación, que haga más prioritaria la inclusión que la alteridad, y que vaya posibilitando una especie de "perijóresis" intrateológica.

J. Martínez Gordo, La fuerza de la debilidad. La teología fundamental de Gustavo Gutiérrez, Ed. Instituto Diocesano de Teología y Pastoral-Desclée de Brouwer, Bilbao, 1994, 357 páginas.

El autor nos ofrece el capítulo segundo de su tesis doctoral sobre las perspectivas -estética, noética y práctica- que han acentuado respectivamente tres gigantes de la teología contemporánea: Balthasar, Pannenberg y Gutiérrez. Las dimensiones elegidas son también tres: el diagnóstico socio-teológico, la teología de la fe y la teología de la revelación. 
Se trata de un análisis crítico y riguroso de la perspectiva práctica o el fundamento teológico, que el teólogo peruano ha construido al hilo de su itinerario biográfico. Nuestro autor detecta en su pensamiento una evolución sin rupturas, dos momentos o períodos teológicos en continuidad, que, calificados asépticamente como GG I y $G G I I$, van a servirle para estructurar en su entorno las claves del pensamiento de Gutiérrez.

El primer capítulo nos ofrece el análisis de su diagnóstico socio-teológico. Recorre su determinación, su descripción y su análisis de causas del hecho mayor latinoamericano - un subcontinente pobre y oprimido, y mayoritariamente cristiano-, sus precisiones sobre el destinatario y el interlocutor de la teología de la liberación - el pobre—, así como la importancia de esta teología entendida como reflexión crítica sobre la praxis.

El segundo presenta su teología de la fe y analiza sus aportaciones sobre la fontalidad del amor de Dios, la fe cristiana y la circularidad de la salvación y de las mediaciones.

El tercero reseña la estructura de su teología de la revelación: la revelación de Dios como misterio, como salvador y liberador, como misterio de amor trinitario, la pobreza y la opresión como lugar de la revelación.

El cuarto lo dedica a la valoración tanto del diagnóstico socio-teológico como de la teología de la fe y de la revelación de $G G$, que organiza a partir de un sencillo esquema: señalar los aspectos que llaman positiva y negativamente la atención. Previamente da cuenta de los prejuicios más comunes (tanto de los más desafectos como de los más apologistas), que su teología suscita, y confiesa -algo que no suele ser muy común- los prejuicios que él mismo compartía al inicio de su investigación. El balance final ofrece una valoración global del itinerario del pensamiento del peruano que supone: la superación crítica del subjetivismo (la prioridad de la Palabra que irrumpe en la existencia humana y la transforma), del premodernismo (la pregunta sobre Dios surge desde el dolor y el sufrimiento del inocente), del pedagogismo (es la obra de un mistagogo), del pelagianismo (Dios como misterio de amor trinitario aparece como el fundamento de todo proceso de liberación), del dogmatismo y de la debilidad noética (su teología articula contextualizadamente las dimensiones ética y noética, la praxis y la razón), y del marxismo (un proyecto liberador integral, incluyente de lo antropológico, estructural y teológico).

La calidad de la obra radica en su capacidad de desentrañar el pensamiento de Gustavo, de desmontar los prejuicios y destacar sus aportaciones al conjunto de la teología. Tiene, por tanto, un valor didáctico indudable. El resultado final de toda su investigación ya lo ha publicado el autor en su libro Dios, amor asimétrico (que recensionamos más adelante) y así podemos valorar con mayor justeza todo su alcance estrictamente teológico. Pero, como muestra de la capa- 
cidad que el pensamiento de $G G$ tiene para quebrar y modificar las preguntas europeas y la del autor para recoger su invitación, cerramos con la trasposición que éste hace a la realidad europea de una de las preguntas más pertinentes del peruano:

¿Cómo hablar de Dios en una sociedad satisfecha que desprecia al pobre y -en la práctica- considera irrelevante a Dios y a todo el discurso sobre El? ¿Cómo hablar de Dios desde el anverso rico y con enormes bolsas de pobreza, defensor de la libertad y dominador de medio mundo, progresivamente increyente e idólatra de sus posibilidades y su bienestar, egoísta y con innegables comportamientos altruistas, ferozmente competitivo y con entrañas de misericordia, generador de injusticia y defensor de la justicia, acaparador y generoso, dionisíaco y prometeico? (pp. 332-333).

J. Martínez Gordo, Dios, amor asimétrico. Propuesta de teología fundamental práctica, Ed. Instituto Diocesano de Teología y Pastoral-Desclée de Brouwer, Bilbao, 1993, 252 páginas.

El autor nos ofrece el resultado final de su investigación sobre Balthasar, Pannenberg y Gutiérrez. Se trata ahora de su propia propuesta de teología fundamental práctica, que resulta de dialogar empática y críticamente con dichos autores, y que, como dice F. Manresa en su presentación, puede resultarintempestiva, como consecuencia de que este proyecto a diferencia de otros al uso concibe la verdad como asimetría y no como correspondencia.

Seguramente aquí se encuentra todo el secreto de una obra que debe ser leída con detenimiento porque muestra no sólo la posibilidad de hacer una teología europea desde la perspectiva de los pobres, sino que muestra además nuevas dimensiones de la realidad de la revelación de Dios y de la fe, cuando son contempladas desde la mirada de los pobres. Se trata —en palabras de su autor- de una propuesta teológica que a las ideologías ilustradas y postmodernas yuxtapone críticamente la ideología de la fraternidad y de la solidaridad en la que estamos radicados, a la que estamos destinados y que se ha anticipado en toda su plenitud en el destino histórico y en la predicación de Jesús de Nazaret.

Su método de empatía trascendental como lo ha calificado B. Forte, da como resultado una propuesta que se extiende a lo largo de 26 tesis, cuyas fomulaciones son ya suficientemente expresivas.

Conviene destacar la primera de ellas porque funciona como horizonte de comprensión de todas las demás. Dice así:

Proposición I: Todas las perspectivas teológicas están fuertemente marcadas por la circularidad hermenéutica que se establece entre lo que se 
considera el núcleo de la revelación y la fe cristiana - por un lado- y el diagnóstico socio-económico, político, cultural y teológico —explícito o implícito- por otro.

Las demás tesis se ordenan en tres apartados:

1. El amor en un mundo radicalmente injusto y asimétrico. Proposiciones referidas al diagnóstico socio-teológico.

Toda propuesta teológica responde implícita o explícitamente a un diagnóstico socio-teológico. Esta propuesta realiza el suyo a través de nueve proposiciones económicas, socio-políticas, socio-culturales y socio-teológicas. Los resultados más notables son:

La identificación del hecho mayor que configura su perpectiva teológica, claramente diferenciado del que funciona en la mayor parte de las teologías al uso (es decir, el ateísmo o la indiferencia):

Proposición 2: Todo diagnóstico socio-religioso se encuentra presidido por un hecho reconocido y vivido como el hecho mayor, como aquél que por su importancia es capaz de marcar y orientar profundamente una perspectiva teológica. El hecho mayor que configura la perspectiva de la propuesta de teología fundamental que se ofrece viene determinado por la creciente primacía que van cobrando en la Europa de los últimos decenios los comportamientos, las actitudes, los planteamientos, las decisiones y las estrategias tanto culturales, como económicas y políticas- que tienden a primar el criterio de la calidad de vida y de la libertad individual al precio —en la prácticade la solidaridad y -sobre todo- de que una minoría mal viva en el interior del sistema europeo, así como que una inmensa mayoría tenga enormes dificultades para subsistir fuera de dicho sistema.

Y la propuesta de relación entre la autorrevelación de Dios y la realidad:

Proposición 13: El diagnóstico de la presente situación socio-teológica conduce a proponer una teología fundamental práctica en la que la relación de la autorrevelación de Dios con la realidad se establezca en términos de crítica continuidad con los resultados que se han alcanzado en el plano antropológico y teológico y de discontinuidad o de muy crítica relación cuando no de ruptura - con la mayoría de los resultados logrados en los planos socio-económico, político y cultural.

2. La revelación de Dios como amor asimétrico. Proposiciones referidas a la teología de la revelación.

Tras dar cuenta de la teología como acto segundo en la explicación razonada de dos proposiciones, se detiene en el despliegue de otras cuatro sobre la trinidad y de una sobre las mediaciones de la revelación. Cabe destacar tres de ellas que relatan y narran trinitariamente su afirmación central de que Dios se revela 
como amor asimétrico:

Proposición 18: El Padre se revela como eterno Silencio y eterno Amante en el Hijo y a partir de El en lo necio, lo débil, lo despreciable y lo inútil de nuestro satisfecho mundo (1Cor 1, 26-31). La propuesta de teología fundamental que se formula pretende señalar —en sintonía con el modo como se revela el Padre- su silenciosa y amorosa compañía en el reverso de nuestra histórica situación. Intenta ser —a la vez - memoria de la fidelidad y gratuidad de dicha presencia - antecedente a todo posible comportamiento solidario- así como explicitación profética de su silencio operante y transformador en el devenir mismo de la historia.

Proposición 19: El Hijo se revela como eterna y amada Palabra asociada libre, gratuita y preferentemente a los condenados por el sistema. La teología fundamental que se propone quiere ser memoria de la compañía de la Palabra de Dios hecha carne - a partir de la cruz y de la resurrección- en los condenados por la historia y por el actual sistema. Intenta ser la voz a través de la que se vaya explicitando el alcance y el significado profético de dicha Palabra, así como del singular comportamiento del eterno Amado en la entraña misma de la historia.

Proposición 20: El Espíritu se revela como eterno Amor en el que se encuentran el silencioso Amante, que es el Padre, y la Palabra Amada, que es el Hijo. La presente propuesta de teología fundamental quiere explicitar la constitutiva inhabitación (síntesis de la radicación y de la destinación en el Espíritu) de los seres humanos en el amor; hacer memoria de la raíz teocéntrica de la existencia y de la historia; denunciar el radical desencuentro económico, político y cultural que se padece, así como recuperar conscientemente la fecundidad del amor divino en la transformación de la presente realidad.

3. La fe como experiencia, razón (teo-logía) y comportamiento asimétricos. Proposiciones referidas a la teología de la fe.

El autor ofrece aquí la justificación y explicitación de una comprensión práctica de la fe desde la perspectiva de los lázaros del sistema. Y nos ofrece primeramente su propia visión de la relación de la fe con la revelación, que es recogida en la siguiente proposición:

Proposición 23: La fe es - a la luz de la trinidad económica- praxis amorosa y solidaria a partir de los lázaros que genera el sistema. Tal praxis se sustenta - antropológicamente - en la radicación - gratuita y matemática - traída y anticipada por el Redentor. Sólo en un momento posterior la fe ha de dar razón de su consistencia veritativa, en crítica referencia con los parámetros epistemológicos vigentes en cada momento histórico. 
Finalmente explicita tres proposiciones sobre la teología de la fe, cuyas formulaciones recogen el carácter agraciado de la experiencia así como sus dimensiones noética, solidaria y práctica.

Proposición 24: La fe es una experiencia - normalmente atemática y silenciosa- de estar radicado y sustentado en el amor-ágape de la trinidad divina. Tal experiencia impulsa a dar gratis lo que gratis se ha recibido ( $\mathrm{Fl} p$ $2,13)$.

Proposición 25: La fe es -igualmente- explicitación tanto de la presencia histórica como de la radicación y destinación en el amor trinitario. En esto consiste la dimensión noética de la fe y la comprensión de la misma como intellectus amoris, como explicitación de la comunicación del amor divino que - a la vez que actúa pluriformemente- se hace presente como compañía, memoria y profecía en la historia y en la propia experiencia de radicación y de destinación en El.

Proposición 26: La fe es - gracias a la acción redentora de la trinidad divina y al auxilio del Espíritu de amor en el que nos encontramos ubicados y por el que estamos inhabitados (Cfr. Col 3,14; 1Tes 3, 12)-praxis solidaria con los demás y, en especial, con los más necesitados.

Unicamente me queda señalar que entre esta obra y la de A. González existen coincidencias notables, que hablan por sí mismas de la afinidad de los tratamientos teológicos que se elaboran desde la perspectiva de los pobres, aunque sus autores vivan geográficamente distantes.

B. Sesboüé, Jesucristo el único Mediador. Ensayo sobre la redención y la salvación. Tomo I. Problemática y relectura doctrinal. Tomo II. El relato de la salvación: Propuesta de soteriología narrativa, Ed. Secretariado Trinitario, Col. Koinonia, Salamanca, 1990 y 1993, 420 y 390 páginas respectivamente.

La obra del jesuita francés es el resultado de un proyecto ambicioso, que, en continuidad con las obras de M. Richard y J. Rivière, da cuenta ordenada del "misterio de la redención". El trabajo está estructurado en tres partes. Las dos primeras han sido recogidas en el primer tomo, y la tercera en el segundo.

Tras señalar algunos aspectos generales, referidos al Salvador y a la salvación, en su primera parte plantea la problemática y el malestar suscitados por algunas de las categorías soteriológicas en las que ha quedado expresada tradicionalmente la fe cristológica en el acontecimiento salvador. Le concede una especial importancia a las críticas provocadas por la "retórica de la sangre", empleada en la interpretación redentora de la cruz. Y trata de ilustrarla por medio de "un florilegio sombrío" de textos sacados de la teología, de la elocuen- 
cia sagrada o de la catequesis (con sabor excesivamente francés).

Tras dar cuenta de las cautelas metodológicas a tener en cuenta para evitar la serie de tergiversaciones históricas padecidas por el vocabulario neotestamentario referido a la fe soteriológica, en la segunda parte realiza un esbozo de historia doctrinal de la soteriología, pasando revista a los términos-clave que la han expresado en la Escritura y a lo largo de la tradición.

Su itinerario recorre invariablemente las siguientes etapas: testimonio de la Escritura, de la tradición y aportación de la teología contemporánea. Los escenarios los constituyen las categorías soteriológicas. Dando preferencia al movimiento descendente de la salvación, las organizará a partir de Cristo Mediador, que considera la referencia primera de la soteriología. En la vía de la mediación descendente estudiará las categorías de Iluminador, Vencedor (Redentor), Liberador, Divinizador, Justicia de Dios. En la vía ascendente, las de Sacrificio, Expiación dolorosa y Propiciación, Satisfacción y Sustitución (Solidaridad). Ambas direcciones encontrarán su síntesis en las categorías de Reconciliación y Perdón.

Todo el segundo tomo - la tercera parte- está dedicado a ofrecer una propuesta propia, titulada El relato de la salvación, y realizada desde la perspectiva de una soteriología narrativa con ambición sistemática. Tras dedicar un capítulo a cuestiones metodólogicas sobre el alcance teológico y la estructura del relato, el ensayo se adentra, a través de una secuencia de tres capítulos, en una relectura narrativa a la luz de Jesucristo de los tres tiempos del relato de la salvación que actúa en nuestra historia: los relatos de la habituación y de la profecía (la salvación en el Antiguo Testamento), los relatos de Jesús (la salvación en el Nuevo Testamento) y los relatos de la Iglesia. Un cuarto capítulo, titulado El relato total: del origen al fin, da cuenta de la dilatación de esta historia hacia el Alfa y la Omega. Cada una de las etapas de este itinerario la concluye con un apartado, de los relatos a las categorías, en el que articula conceptulamente "los efectos de sentido" de los relatos.

El trabajo es serio y riguroso. Sin duda llena un vacío que existía en la teología, después de la floración de obras cristológicas de la década de los setenta y comienzos de los ochenta, en la que B. Sesboüé también participó. Una vez compuesta de nuevo la unidad entre cristología y soteriología, se hacía necesario abordar críticamente las categorías soteriológicas en su sentido estricto. Parecía también que era un tiempo oportuno para aceptar el reto de la propuesta realizada por J. B. Metz acerca de una soteriología narrativarememorativa. Aporta además en directo datos de la soteriología patrística, y sintetiza las mejores aportaciones de la teología contemporánea. De su ensayo de soteriología narrativa hay que destacar las categorías que ofrece para comprender la causalidad de la salvación: las parejas "seducción"-"conversión" y "contagio"-"fe" como pertenecientes a la estructura comunicativa de la salva- 
ción. Sin embargo, es necesario hacerle tres observaciones críticas.

Resulta sorprendente que en una obra de semejante magnitud solamente se recoja en dos ocasiones la aportación de G. Gutiérrez entre los teólogos de la liberación. ¿Qué puede significar tanta ausencia y semejante desconocimiento en una obra que, de acuerdo con los cánones más exigentes de la comunidad científica, acarrea un enorme caudal de información rigurosa desde las fuentes vetero y neotestamentarias hasta las aportaciones de los teólogos y pensadores modernos y contemporáneos, pasando por la patrística y la teología medieval? ¿Cómo explicar satisfactoriamente que un ensayo sobre la redención y la salvación no dedique más que esas breves referencias en un total de casi ochocientas páginas a una teología como la de la liberación cuya perspectiva es soteriológica? ¿Acaso la teología de la liberación no ha hecho ninguna aportación merecedora de consideración en relación con la doctrina de la redención, al margen de la titulación de Jesucristo como Liberador? Si en cada categoría soteriológica que revisa, y lo hace nada menos que con once, abre un apartado que da cuenta del tratamiento que la misma ha recibido en la teología contemporánea, ¿qué explicación se puede dar al hecho de que no recurra en ninguna ocasión a teólogos como L. Boff, J. L. Segundo, I. Ellacuría, J. Sobrino?

El nudo gordiano de la tarea teológica en orden a recuperar la legitimidad cristiana del discurso soteriológico no consiste en el simple hecho de priorizar el movimiento descendente de la salvación de Dios frente al movimiento ascendente, tal y como se hizo durante el primer milenio. En mi opinión lo decisivo sólo ocurre cuando esta soteriología "desde arriba" llega hasta la kenosis, y para ello debe tener claro que lo realmente decisorio para constituirse en logos sobre la revelación de la salvación cristiana acaece cuando se construye desde los de abajo, aunque obviamente este modo de proceder no evite los malestares contemporáneos, sino que los acreciente en forma de otros rechazos y de otras contestaciones, que en ocasiones y lugares determinados llegan hasta el estallido de la violencia, la persecución y el martírio. B. Sesboüé no tiene para nada en cuenta esta perspectiva de los pobres, y, desde su tratamiento de Jesucristo Mediador, esta enorme laguna convierte su ensayo sobre la redención y la salvación en excesivamente ahistórico.

Esta ahistoricidad aparece con mayor claridad en su propia propuesta de soteriología narrativa. Casi en la última página de su obra escribe refiriéndose a la seducción provocada por el amor kenótico de Dios: "Esta seducción se hace contagio en la Iglesia, a través de la cadena de testigos encargados de anunciar el Evangelio y de invitar a la fe" ( T. II, p. 363). Pues bien, a pesar de esto no ha sentido la necesidad de recordar algún relato contemporáneo contagioso de salvación a lo largo de todo su ensayo. Leyendo el libro uno tiene la impresión de que las historias de salvación que merecen ser narradas y recordadas, terminaron con las consignadas en las Escrituras. Obviamente no se puede negar la 
normatividad de éstas, pero pienso que entre los relatos fundacionales del cristianismo y las historias humanas y cristianas que, por ser buena noticia son relatos actualizados de salvación, se establece una relación hermenéutica. Los relatos de las historias fundacionales permiten percibir lo salvífico de las historias actuales y éstas permiten captar mejor lo histórico de aquellos relatos de salvación. A mi entender sólo así se cumple satisfactoriamente con la propuesta metziana de una teología rememorativa y narrativa capaz de superar las insuficiencias de una teología de la salvación puramente argumentativa.

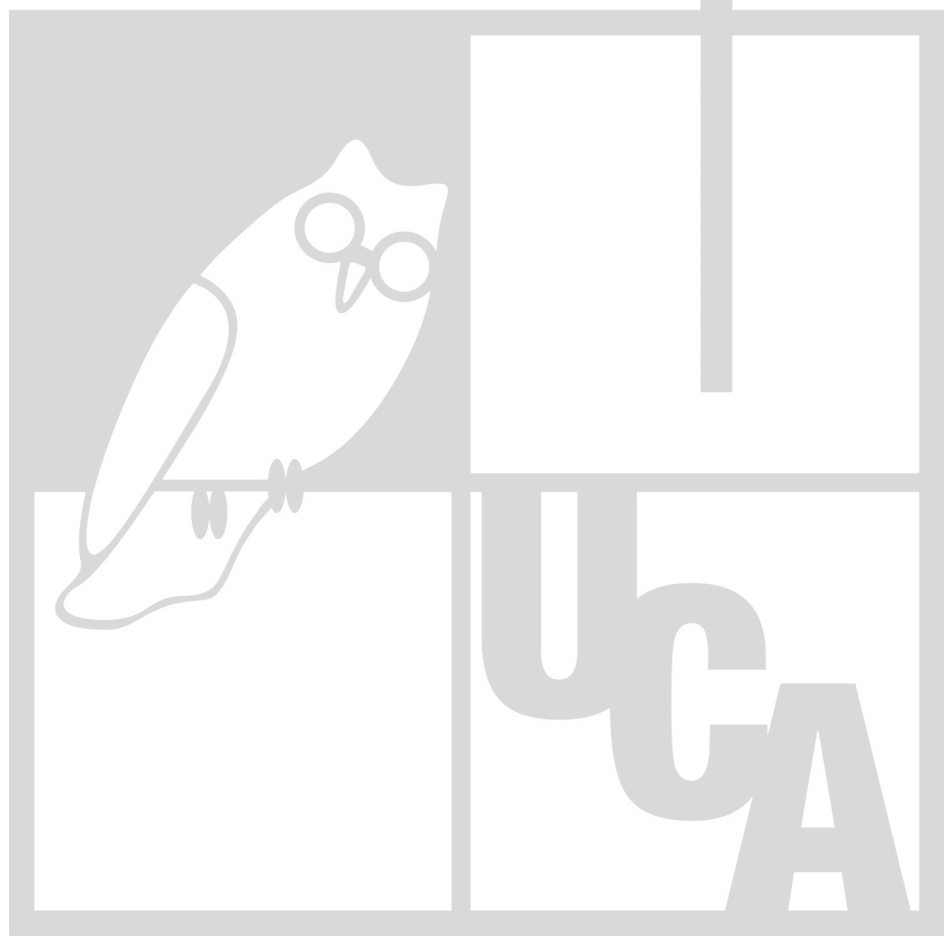

